\title{
Transient Left Bundle Branch Block in the Setting of Hyperkalemia
}

\author{
Abida Naz ${ }^{1}$, Tikal Kansara $^{1}$, Marco Ruiz Santillan ${ }^{2}$, Mohammad Saeed ${ }^{1}$ \\ 1. Internal Medicine, New York Medical College, NYC Health + Hospitals/Metropolitan, New York, USA 2. Internal \\ Medicine, New York Medical College, Metropolitan Hospital Center, New York, USA
}

Corresponding author: Mohammad Saeed, saeedtg2003@yahoo.com

\begin{abstract}
Hyperkalemia is a potentially life-threatening condition that can lead to sudden cardiac death. We report a case of transient left bundle branch block (LBBB) pattern on an electrocardiogram (EKG) secondary to hyperkalemia in a patient with a history of end-stage renal disease. A 48-year-old female presented to the emergency department (ED) with chief complaints of weakness and shortness of breath after a missed hemodialysis session. A 12-lead EKG in the ED showed the LBBB pattern with left axis deviation, prolonged PR interval, and peaked T-waves in the precordial leads. The initial serum potassium level was $8.5 \mathrm{mEq} / \mathrm{L}$. EKG changes resolved after correcting the serum potassium level.
\end{abstract}

Review began 05/30/2021 Review ended 06/28/2021 Published 07/24/2021

\section{() Copyright 2021}

$\mathrm{Naz}$ et al. This is an open access article distributed under the terms of the Creative Commons Attribution License CC-BY 4.0., which permits unrestricted use, distribution, and reproduction in any medium, provided the original author and source are credited.
Categories: Cardiology, Emergency Medicine, Nephrology

Keywords: left bundle branch block, severe hyperkalemia, myocardial infarction, hemodialysis, hyperkalemiainduced ekg changes

\section{Introduction}

Hyperkalemia is a frequent clinical problem most often caused by impaired urinary potassium excretion secondary to acute kidney injury or chronic kidney disease. Severe hyperkalemia is a medical emergency requiring urgent interventions and can lead to life-threatening cardiac arrhythmias if left untreated. Although serum potassium concentration is the most reliable method for determining the severity of hyperkalemia, electrocardiogram (EKG) changes associated with hyperkalemia may suggest the diagnosis before conducting a blood test. Although various EKG changes may be associated with hyperkalemia, peaked T-waves in the precordial leads are usually the first and most commonly recognized findings. Cardiac conduction abnormalities have also been reported with hyperkalemia at severely elevated levels $[1,2]$. Here, we report a case of transient left bundle branch block (LBBB) secondary to hyperkalemia in a patient with a history of end-stage renal disease (ESRD).

\section{Case Presentation}

A 48-year-old female with ESRD on hemodialysis presented to the emergency department (ED) with complaints of generalized weakness and shortness of breath after she missed one hemodialysis session. On arrival to the ED, her heart rate was 110 beats per minute, blood pressure was $210 / 90 \mathrm{mmHg}$, respiratory rate was 22 breaths per minute, and oxygen saturation was $90 \%$ on $2 \mathrm{~L}$ of oxygen. General and systemic examinations were suggestive of volume overload. The laboratory analysis showed severe hyperkalemia with a serum potassium level of $8.5 \mathrm{mEq} / \mathrm{L}$, blood urea nitrogen of $118 \mathrm{mg} / \mathrm{dL}$, creatinine of $13 \mathrm{mg} / \mathrm{dL}$, sodium of $134 \mathrm{mEq} / \mathrm{L}$, chloride of $99 \mathrm{mEq} / \mathrm{L}$, bicarbonate of $22 \mathrm{mmol} / \mathrm{L}$, and calcium of $9.4 \mathrm{mg} / \mathrm{dL}$. Urine toxicology was negative. Her initial EKG showed a heart rate of 92 beats per minute, PR interval of $222 \mathrm{ms,} \mathrm{QRS} \mathrm{duration} \mathrm{of}$ $174 \mathrm{~ms}$, dominant S-wave in V1, broad monophasic R-wave in lateral leads, absence of Q-wave in lateral leads (I, aVL, V5-V6), and left axis deviation, suggestive of LBBB (Figure 1). On review of records, a normal baseline EKG with no structural heart disease was noted. Although new-onset LBBB could have been due to myocardial infarction/ischemia or hyperkalemia, due to a history of ESRD with missed hemodialysis, we treated this as a case of severe hyperkalemia which was confirmed with serum potassium level. 


\section{Cureus}

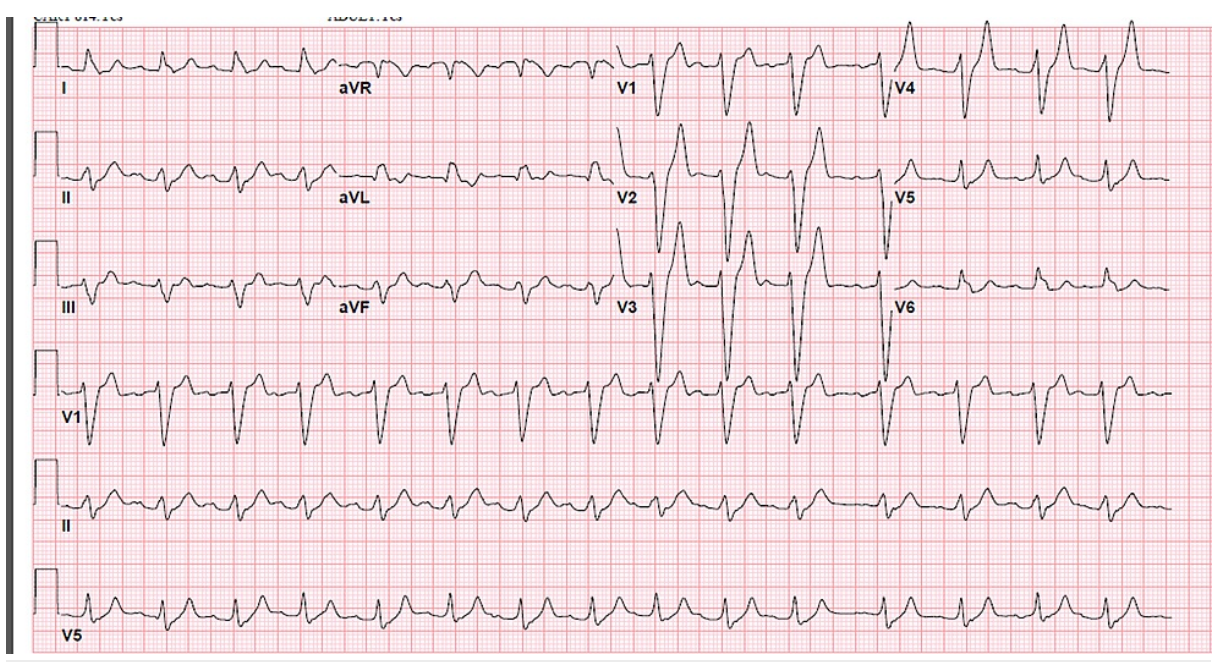

FIGURE 1: Initial EKG.

First-degree atrioventricular block (PR: $222 \mathrm{~ms}$ ), LBBB (QRS: $174 \mathrm{~ms}$ ), and tall tented T-waves.

EKG: electrocardiogram; LBBB: left bundle branch block

She was initially treated with intravenous calcium gluconate, insulin with glucose, albuterol nebulization, and intravenous furosemide (Figure 2). Hemodialysis was done on an urgent basis after which potassium level improved to $5.5 \mathrm{mEq} / \mathrm{L}$ and her shortness of breath resolved. A repeat EKG showed normal sinus rhythm (Figure 3).

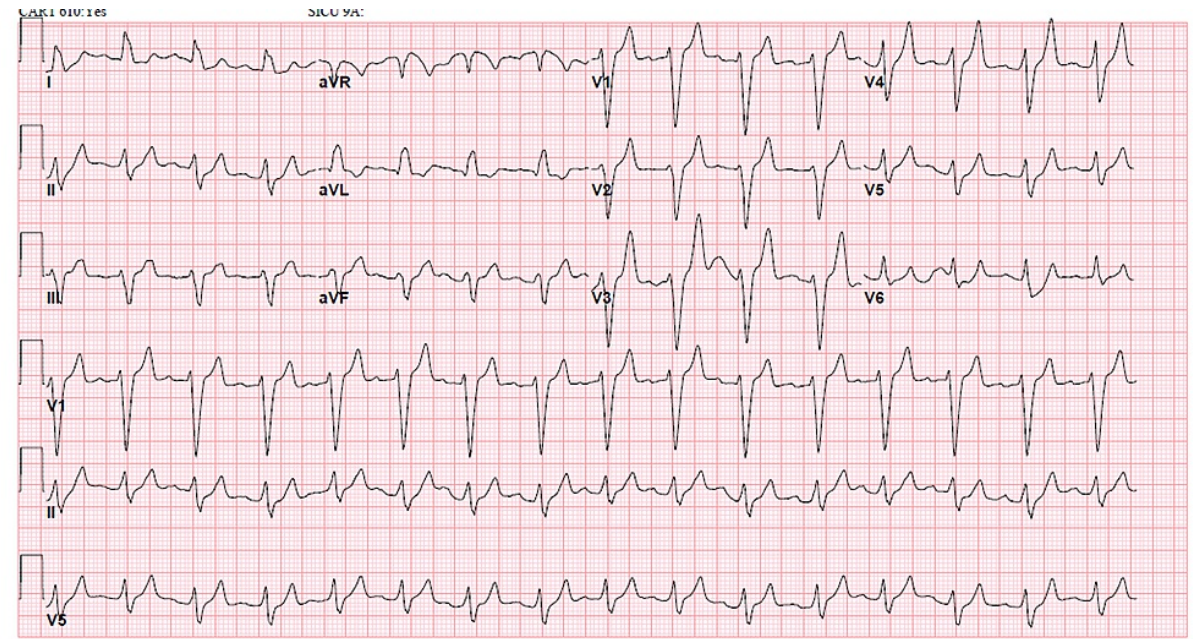

\section{FIGURE 2: EKG after intravenous calcium gluconate.}

EKG after calcium gluconate showed shortening of the PR interval (202 ms from $222 \mathrm{~ms}$ ) and mild narrowing of the QRS complex (158 ms from $174 \mathrm{~ms}$ ).

EKG: electrocardiogram 


\section{Cureus}

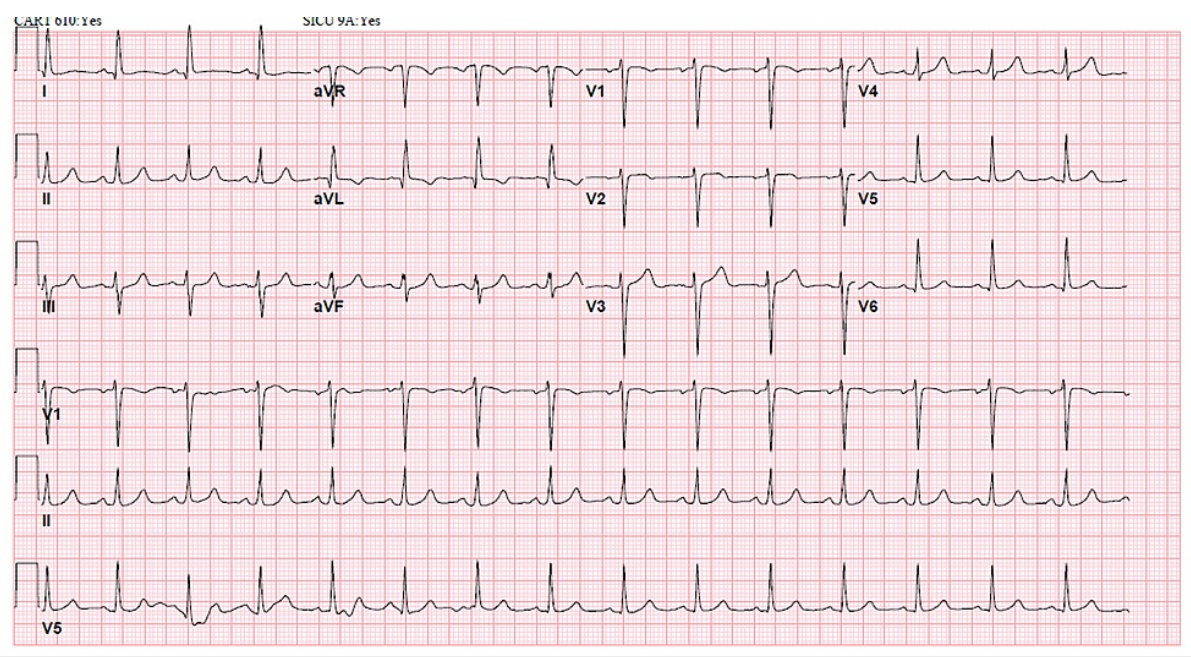

FIGURE 3: EKG after hemodialysis showing normal sinus rhythm.

EKG: electrocardiogram

In the setting of ESRD and absence of known precipitating factors for increased serum potassium level, her hyperkalemia was attributed to ESRD. On normal cardiac evaluation including an exercise stress test, her EKG changes were assumed to be related to hyperkalemia which resolved after hemodialysis.

\section{Discussion}

Hyperkalemia may be associated with EKG changes. However, EKG findings in hyperkalemia are seen in only $50-64 \%$ of patients with potassium levels of more than $6.5 \mathrm{mEq} / \mathrm{L}$ [3]. Sequential EKG changes noted with rising potassium levels include peaked T-waves, prolonged PR interval, prolonged QRS, loss of P-wave, escape rhythms, sine wave configuration, ventricular fibrillation, and pulseless activity or asystole. These EKG changes can be explained by the physiological effect of potassium on myocardial cells. Hyperkalemia decreases the resting membrane potential (RMP), the magnitude of the action potential, and the maximum rate of increase of phase 0 in the cardiac muscle. A high concentration of extracellular potassium slows impulse conduction through all cardiac tissue, accounting for numerous EKG findings. The effect of hyperkalemia depends on the tissue involved, with the atrial myocardium being the most sensitive, the ventricular myocardium less sensitive, and the specialized tissue (sinoatrial node and His bundle) the least sensitive.

Mild-to-moderate hyperkalemia causes depression of conduction between adjacent cardiac myocytes, resulting in the prolongation of the PR and QRS intervals as potassium levels increase. P-wave amplitude disappears early because of the sensitivity of atrial myocytes to hyperkalemia. As the severity of hyperkalemia increases, the sinoatrial and atrioventricular conduction is further suppressed, resulting in the appearance of escape beats and escape rhythms. The QRS complex continues to widen and may blend with the T-wave, creating a sine wave appearance in the EKG. If the potassium level is allowed to rise without treatment, it can cause ventricular fibrillation.

The presence of these EKG findings often guides on how aggressively to treat hyperkalemia [4]. However, there have been reports of normal EKG even with serum potassium levels greater than $10 \mathrm{mEq} / \mathrm{L}$ [5]. Adverse outcomes of hyperkalemia include symptomatic bradycardia, ventricular tachycardia, ventricular fibrillation, cardiac arrest, and death. According to one study, the median time from EKG to an adverse event was 47 minutes [4]. Hence, it becomes necessary to treat the patient in the ED as soon as EKG changes are suspicious of hyperkalemia. Previous studies have reported rate-dependent LBBB, wherein the EKG did not show any changes at a lower rate; however, as the heart rate increased, atrial fibrillation with LBBB was seen in a patient with hyperkalemia [6]. As LBBB resolved in our patient after hemodialysis, and cardiac evaluation including exercise stress test was normal, LBBB can be attributed to hyperkalemia.

Severe hyperkalemia with a potassium level of $>6.5 \mathrm{mEq} / \mathrm{L}$ is regarded as a medical emergency and the patient should receive cardiac monitoring in a controlled setting with immediate medical interventions. If any EKG changes are noted, the patient should be promptly administered membrane stabilizers such as intravenous calcium and cellular potassium uptake agents. This should be followed as soon as possible by therapies to remove potassium from the body, including hemodialysis, diuretics, and gastrointestinal cation exchange resins [7].

\section{Conclusions}


Our case illustrates the effect of serum potassium on EKG which an alert physician can recognize to provide lifesaving treatment even before receiving biochemical confirmation.

\section{Additional Information \\ Disclosures}

Human subjects: Consent was obtained or waived by all participants in this study. Conflicts of interest: In compliance with the ICMJE uniform disclosure form, all authors declare the following: Payment/services info: All authors have declared that no financial support was received from any organization for the submitted work. Financial relationships: All authors have declared that they have no financial relationships at present or within the previous three years with any organizations that might have an interest in the submitted work. Other relationships: All authors have declared that there are no other relationships or activities that could appear to have influenced the submitted work.

\section{References}

1. Littmann L, Gibbs MA: Electrocardiographic manifestations of severe hyperkalemia. J Electrocardiol. 2018, 51:814-7. 10.1016/j.jelectrocard.2018.06.018

2. Diercks DB, Shumaik GM, Harrigan RA, Brady WJ, Chan TC: Electrocardiographic manifestations: electrolyte abnormalities. J Emerg Med. 2004, 27:153-60. 10.1016/j.jemermed.2004.04.006

3. Freeman K, Feldman JA, Mitchell P, et al.: Effects of presentation and electrocardiogram on time to treatment of hyperkalemia. Acad Emerg Med. 2008, 15:239-49. 10.1111/j.1553-2712.2008.00058.x

4. Durfey N, Lehnhof B, Bergeson A, et al.: Severe hyperkalemia: can the electrocardiogram risk stratify for short-term adverse events?. West J Emerg Med. 2017, 18:963-71. 10.5811/westjem.2017.6.33033

5. Khattak HK, Khalid S, Manzoor K, Stein PK: Recurrent life-threatening hyperkalemia without typical electrocardiographic changes. J Electrocardiol. 2014, 47:95-7. 10.1016/j.jelectrocard.2013.07.012

6. Fransen NE, de Vos L, van Kesteren HA: Rate-dependent left bundle branch block caused by hyperkalaemia . Neth Heart J. 2016, 24:217-8. 10.1007/s12471-015-0795-1

7. Dépret F, Peacock WF, Liu KD, Rafique Z, Rossignol P, Legrand M: Management of hyperkalemia in the acutely ill patient. Ann Intensive Care. 2019, 9:32. 10.1186/s13613-019-0509-8 